\title{
Parasitismo Humano por Carrapatos na Mata Atlântica, Rio de Janeiro, Brasil
}

\author{
Nicolau Maués Serra-Freire ${ }^{\circledR}$, Laura Morena Mendes de Sena ${ }^{2}$ \& Ana Beatriz Pais Borsoi ${ }^{1}$
}

1. Fundação Oswaldo Cruz, e-mail: nmserrafreire@gmail.com (Autor para correspondência ${ }^{\bowtie}$ ), anaborsoi@gmail.com. 2. Universidade Estácio de Sá, e-mail:nmsf@ioc.fiocruz.br.

\author{
EntomoBrasilis 4 (2): 67-72 (2011)
}

Resumo. Entre janeiro de 1997 e dezembro de 2007 foi realizada uma investigação epidemiológica objetivando identificar casos de parasitismo por carrapatos em humanos na comunidade que invadiu e está ocupando Área de Proteção Ambiental - (APA), entre o "Parque Estadual do Maciço da Pedra Branca" e o "Parque Nacional da Floresta da Tijuca" na cidade do Rio de Janeiro, RJ. Foram comprovados 6.072 casos de ixodidose, relacionados às espécies: Amblyomma aureolatum (Pallas), Amblyomma cajennense (Fabricius), Amblyomma dubitatum Neumann, Amblyomma ovale Kock, Amblyomma varium Kock, Ixodes loricatus Neumann e Rhipicephalus sanguineus (Latreille) em relação parasitária com moradores do espaço trabalhado. Foram calculados indicadores de saúde relacionados ao coeficiente de prevalência, índice de abundância, coeficiente de dominância, intensidade média de parasitismo, e coeficiente de correlação entre o parasitismo e o sexo, faixa etária, atividade social dos infectados, o risco relativo, e o risco atribuível para o parasitismo, e desenvolvida estatística descritiva para visualização do fenômeno, e estatística analítica dos dados para dar suporte às conclusões. Foi salientada a importância destas espécies de ixodídeos na transmissão de patógenos, bioagentes de doenças infecciosas reincidentes, ou de recém introdução no espaço geopolítico brasileiro.

Palavras-Chave: Carrapato; humano; Parque Estadual da Pedra Branca; Ixodidae

\section{Human Parasitism for Ticks in the Atlantic Forest, Rio de Janeiro, RJ, Brazil.}

Abstract. From January 1997 to December 2007 was carried out a epidemiologic investigation to identify a cases of parasitism in the human community that is invaded and occupied area of Environmental Protection - (BB), between the "State Park of the Pedra Branca" and "National Park of Tijuca Forest" located in Rio de Janeiro, RJ, Brazil. These work comprouved 6,072 cases of ixodidosis in relation with species: Amblyomma aureolatum (Pallas), Amblyomma cajennense (Fabricius), Amblyomma dubitatum Neumann, Amblyomma ovale Kock, Amblyomma varium Kock, Ixodes loricatus Neumann and Rhipicephalus sanguineus (Latreille) infected local human living into the space worked. Through an analysis of statistical data demonstrating transcribes the importance of transmission of pathogens and serves as a model for understanding of the human $\mathrm{X}$ tick.

Keywords: Human bean; Ixodidae; Pedra Branca State Park; Tick

$\mathbf{O}$ Parque Estadual do Maciço da Pedra Branca e o Parque Nacional da Floresta da Tijuca têm sido alvos de pesquisas em decorrência do desrespeito social com sucessivas invasões e depredações do ambiente natural. Nestes ambientes ainda há resquício da Mata Atlântica com flora primitiva que vem sendo agredida por cortes e/ou queimadas, além do desafio à flora e fauna por introdução de espécies exóticas ao local, inclusive a colonização humana. Assim é que carrapatos de animais silvestres têm maior probabilidade de parasitarem humanos.

Os carrapatos são cosmopolitas e estão amplamente dispersos no Brasil (BARRos-Batesti et al. 2006), podendo atuar como vetores de bioagentes de doenças (Sonenshine 1991; Estrada-Peña \& Jongejan 1999), e por si só exercer diversos efeitos deletérios no organismo do hospedeiro, que vão desde a lesão cutânea, à anemia ocasionada por uma infestação maciça, à inoculação de toxinas neurotrópicas que causam paralisia flácida ascendente (SERRa-Freire 1983), paralisias setoriais, e eventualmente induzir à morte. Obviamente, tais efeitos variam conforme a espécie de carrapato e a área geográfica.

A grande maioria das espécies de Ixodida (cerca de 80 - 90\%) parasita exclusivamente animais silvestres; a minoria restante $(20-10 \%)$ pode ser encontrada parasitando os animais domésticos e o homem. Assim é que a pesquisa sobre carrapatos é expressivamente maior em número, no direcionamento para as espécies de maior impacto econômico. Por outro lado, o conhecimento das espécies parasitas de animais silvestres torna-se relevante, já que muitas delas participam diretamente na manutenção enzoótica de patógenos. Além disso, a história mostra que algumas destas espécies, antes confinadas ao ambiente silvestre, são vetores de bioagentes de zoonoses emergentes (Labruna 2004).

No ambiente o carrapato procura locais protegidos, sombrios e com pouca ventilação, pode ser parte da biomassa (árvores, arbustos, vegetação rasteira), do ecótopo (buracos no solo, fendas em rochas, torrões de solo arado), ou já em ambiente antrópico (paredes de casas humanas, muros de limitação entre domínios urbanos, falhas no assentamento de piso interior ou exterior de habitações), e mesmo estruturas dentro da habitação ocupada por humanos (móveis, sancas, cortinas, quadros de parede). Nestes ambientes protegidos as teleóginas realizam as posturas dos ovos, ou os estádios ingurgitados fazem mudas e ecdises. As larvas emergidas da eclosão dos ovos, e os novos estágios conseqüentes às ecdises saem dos paradeiros à procura de hospedeiro para se alimentar. Fixam-se no hospedeiro, praticam o hematofagismo com maior freqüência em regiões de pele mais fina, úmidas, com circulação abundante e de difícil acesso para o animal se autolimpar (SERRA-Freire 2001).

A família Ixodidae engloba o maior número de espécies 
de carrapatos, com 13 gêneros e 692 espécies dispersas no mundo (NAVA et al. 2009), formando dois grandes grupos, que reúne cinco subfamílias (HoogstraAl \& AEschlimann 1982; ONoFrio et al. 2006): Grupo Prostriata, Subfamília Ixodinae; e Grupo Metastriata com quatro Subfamílias: Amblyomminae, Haemaphysalinae, Hyalomminae) e Riphicephalinae. FILIPPOvA (1984) considera apenas duas Subfamílias: Ixodinae (Prostriata) e Amblyomminae (Metastriata) (Oliver Jr. 1989).

Osixodídeos fixam-senohospedeiro, nelepermanecendo por períodos mais ou menos prolongados, e isso ocorrendo nos três estádios do ciclo vital (larvas, ninfas e adultos), realizando um único, porém longo repasto em cada estágio parasitário (SERRA-Freire 2001). A postura dos ovos ocorre depois da fêmea fecundada ingurgitar, quando então abandona o hospedeiro; portanto, os ovos são postos fora do hospedeiro e em esconderijos. A postura é única e o número de ovos é grande. Esse elevado número está relacionado com a sobrevivência da espécie, pois os ixodídeos são predados por diferentes animais; as teleóginas são alimento de aves [garça (Ardea alba L., gaviãozinho carrapateiro ou chimango (Milvago chimango Vieillot), galinha d'angola (Numida meleagridis L.)], e as larvas por aranhas e formigas, e ainda podem desidratar com facilidade se ficarem isoladas. A amplitude do ciclo biológico é muito variável, de 3 a 12 meses, e os adultos podem sobreviver por 5 a 12 anos, incluindo longos períodos de jejum (Flechtmann 1973; Serra-Freire \& Miziara, 1989).

Do ponto de vista médico veterinário são artrópodes de muita importância por produzirem injúrias diretas, e viabilizarem a manutenção de várias enzootias. Por serem dentre os Acari aqueles de maior porte físico, e por serem fáceis de encontrar em virtude de seus hábitos parasitários, os carrapatos têm sido mais estudados em relação aos demais Acari (FlechtMAnN 1973). Os carrapatos estão entre os principais vetores de microorganismos causadores de doenças (vírus, bactérias, protozoários, riquétsias e fungos) para os animais e o homem. Nos carrapatos, os patógenos podem ser transmitidos de um estágio para o outro (transmissão transestadial: de larvas para ninfa, ou de ninfa para adulto) e/ ou entre gerações (transmissão transovariana de uma fêmea para seus ovos), fazendo dos carrapatos muitas vezes, grandes reservatórios de patógenos na natureza (LABRUNA 2004). A saliva dos carrapatos contém quinases que catalisam a bradicinina, o que explica em parte a ausência de dor. As prostaglandinas presentes na saliva inibem a produção de interleucina 1 e a migração de macrófagos (MASSARD \& FonSECA 2004).

Considerando que o conhecimento da situação da saúde na população é fundamental para o planejamento, a tomada de decisão, e a conseqüente implementação de ações em saúde, onde várias espécies de carrapatos atuam como vetores, por exemplo, de Rickettsia rickettsii causadora da Febre Maculosa Brasileira, tanto por espécies tidas como ectoparasitos de animais silvestres (ARAgÃo 1936; Lemos et al. 1996; Souza et al. 2006; GaZÊTA et al. 2009), como de animais domésticos (Boero 1957; Barros-Batesti et al. 2006; PINTER et al. 2008; CUNHA et al. 2009), é necessário conhecer a dimensão da ixodofauna que infecta humanos.

Nestas condições este trabalho tem como objetivo registrar o parasitismo de humanos por carrapato (Acari: Ixodidae) em uma Área de Proteção do Ambiente na região Metropolitana do Rio de Janeiro, a partir de um inquérito sobre os riscos a que vem se expondo diuturnamente os humanos dessa comunidade que invadiu e está ocupando Área de Proteção Ambiental (APA) do Maciço da Pedra Branca e da Tijuca, e que poderá servir como modelo para o entendimento da relação carrapato $\mathrm{x}$ humano.

\section{MATERIAL E MÉTODOS}

A pesquisa teve delineamento de aglomerado, observacional, transversal, caracterizada como estudo ecológico, trabalhando com a população ajustada em relação aos anos de investigação, para efeito dos cálculos dos indicadores.
Área e período de investigação. O estudo foi desenvolvido no espaço geográfico dos Maciços da Pedra Branca, e da Tijuca, na área administrada pelo Instituto Brasileiro do Meio Ambiente (IBAMA) e Instituto Estadual de Floresta (IEF) (Figura 1).

O período total de investigação foi Janeiro/1997 a Dezembro/2007, desenvolvido em duas etapas: a primeira entre Janeiro de 1997 e Junho de 2005, foi desenvolvida de forma menos intensa; quando se buscou o conhecimento da comunidade alvo, procurando interagir com ela para conseguir a confiança e colaboração para execução da segunda etapa, esta mais intensiva. Nesta etapa, com a autorização dos órgãos competentes de natureza estatal, procurou-se a colaboração de organização social local, e de liderança nas populações humanas integrantes do grande complexo habitacional deste espaço político-administrativo. Na segunda, entre Julho de 2005 e Dezembro de 2007, intensificou-se a procura dos casos, sendo o tamanho final da amostra correspondeu a $1 \%$ da população estimada do número de pessoas moradoras do complexo, sem distinção da faixa etária, origem étnica, peso, altura, credo, afinidade esportiva, política e musical, condição de trabalho e renda, ocupação social e escolaridade. O critério para inclusão na amostragem foi o da permissão do exame físico, resposta ao questionário, e autorização para uso dos dados para fins científicos. A amostragem foi por sorteio em cacho dentre os grupos que formavam os núcleos.

Captura e processamento de Carrapatos. Os carrapatos encontrados no corpo dos humanos foram removidos por torção contínua em torno do próprio eixo antero-posterior do idiossoma até o desprendimento da pele. Os espécimes removidos foram mortos por imersão em água quente $\left( \pm 70^{\circ} \mathrm{C}\right)$ e preservados em etanol $70^{\circ} \mathrm{GL}$, e transportados até o Laboratório de Ixodides Referência Nacional para Vetores das Riquétsias, do Instituto Oswaldo Cruz, FIOCRUZ, no Rio de Janeiro, RJ, Brasil.

A ficha questionário padrão usada para levantar os dados com cada pessoa integrante da amostra, continha perguntas relacionadas à identificação da pessoa; ao habitat que ocupava com detalhamento do espaço geográfico, geopolítico e políticoadministrativo; à função na sociedade; e aos tipos de atividades que participou na comunidade, nos últimos três dias antes da percepção da presença do carrapato parasitando.

Em laboratório os carrapatos foram triados por estereomicroscopia, de acordo com o gênero, estádio e integridade física. A identificação de ninfas e adultos, com uso de estereomicroscópio, foi processada com uso de chaves dicotômicas (Robinson et al. 1926; CoOley \& Kohls 1944; Clifford \& Anastos 1960; Aragão \& FonseCa 1961; Jones et al. 1972; FleChtMAnn 1973, Keirans \& Durden 1998; Barros-Battesti et al. 2006).

As larvas foram examinadas por microscopia de luz com uso de chave dicotômica de AMOrim \& SERra-Freire (1999), ClifFord \& ANASTOS (1960) e BARBIERI (2005).

Os dados das espécies identificadas e dos estádios encontrados foram trabalhados por estatística demonstrativa, com ênfase na tendência anual e secular, e analítica por medidas de posição (média e desvio padrão), e por cálculo de coeficientes de similaridades (CSs), coeficiente de dominância, de associação e incidência; as diferenças entre coeficientes para os sexos foram testadas pelo teste z, com significância arbitrada em 5\% (SERRAFREIRE 2002).

\section{RESULTADOS E DISCUSSÃO}

Durante as duas etapas do estudo, período onze anos, foi constatado que Amblyomma cajennense (Fabricius) foi a espécie mais freqüente no parasitismo humano com 4.495 casos (Tabela 1). Também foi a espécie dominante entre os 12.351 fixados na pele (Tabela 1). Dentre o total de humanos parasitados por carrapatos, os homens estavam mais infectados $(56,4 \%)$ em relação às mulheres $(43,6 \%)$, sendo que diferenças foram constatadas em todas as classes de faixa etária (Figura 2). Encontros de outras espécies de carrapatos duros parasitando 


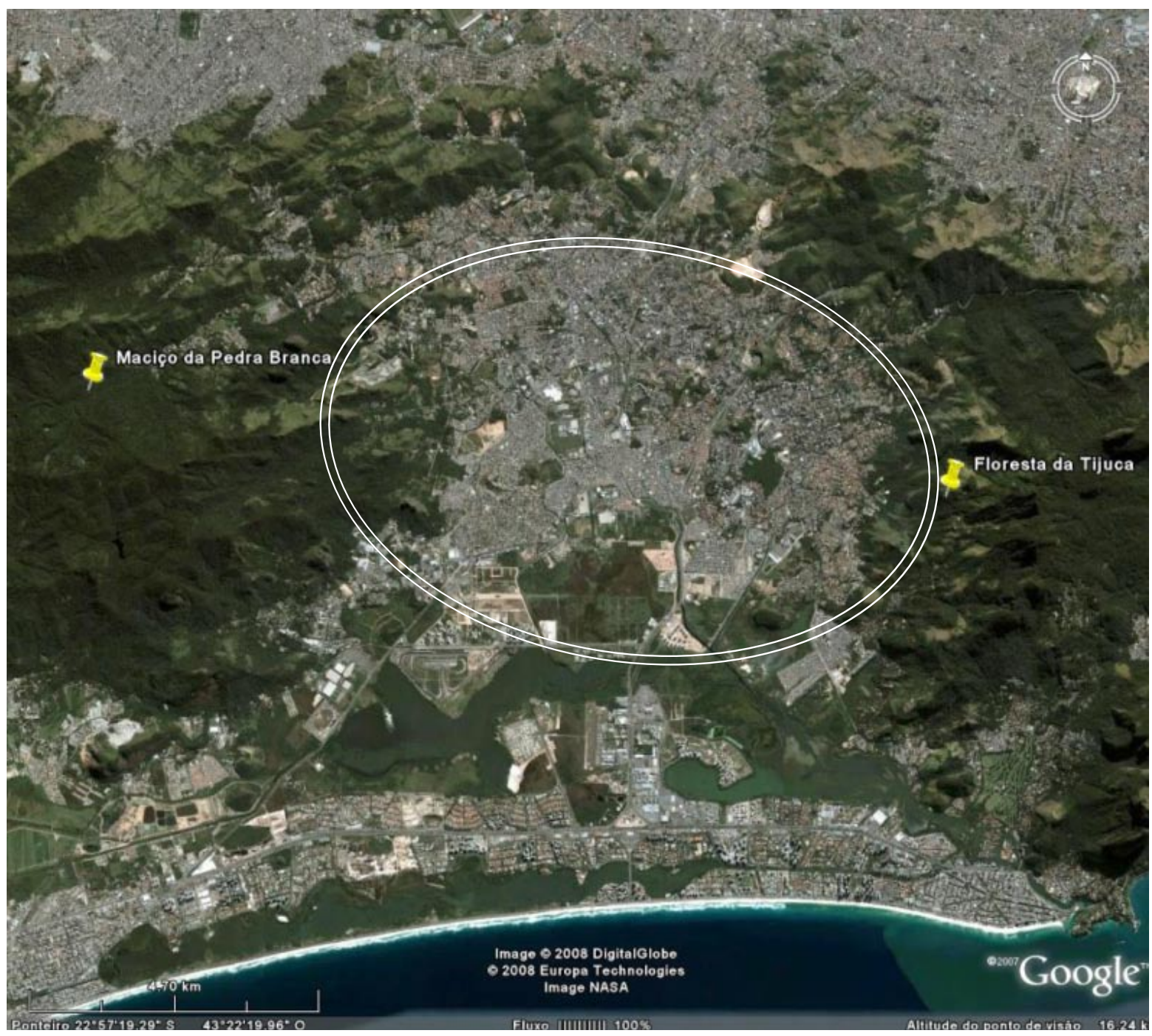

Figura 1. Caracterização aeroespacial do Maciço da Pedra Branca, com visão da área de invasão humana no Parque Nacional da Tijuca - Floresta da Tijuca, em 2007, espaço de estudo sobre infecção de humanos por carrapatos (Fonte: Google).

Tabela 1. Relação entre as espécies de carrapatos duros (Acari: Ixodidae) pelo coeficiente de dominância (CD), e delas com humanos hospedeiros, nas comunidades do Maciço da Pedra Branca e do Maciço da Tijuca, constatado durante pesquisa entre Janeiro/1997 e Dezembro/2007.

\begin{tabular}{|c|c|c|c|c|c|}
\hline \multicolumn{3}{|c|}{ Carrapato } & \multicolumn{3}{|c|}{$\begin{array}{c}\text { Humano hospedeiros de carrapatos, sem (com) parasitismo } \\
\text { simultâneo por mais de uma espécie }\left(\mathrm{N}^{\circ}\right)\end{array}$} \\
\hline Espécie & Número & CD (\%) & Total & Homem & Mulher \\
\hline Amblyomma aureolatum & 176 & 1,43 & $176(20) a$ & $102(12) \mathrm{a}$ & $74(8) a$ \\
\hline Amblyomma cajennense & 8.668 & 70,18 & $4.423(79) \mathrm{b}$ & $2.649(35) \mathrm{b}$ & $1.774(44) b$ \\
\hline Amblyomma dubitatum & 201 & 1,63 & $201(8) c$ & $96(3) \mathrm{c}$ & $105(5) c$ \\
\hline Amblyomma ovale & 52 & 0,42 & 46 & 24 & 22 \\
\hline Amblyomma varium & 675 & 5,47 & 528 & 302 & 226 \\
\hline Ixodes loricatus & 9 & 0,07 & 9 & 7 & 2 \\
\hline Rhipicephalus sanguineus & 2.570 & 20,80 & $640(51) \mathrm{d}$ & $214(20) d$ & $426(31) \mathrm{d}$ \\
\hline Soma & 12.351 & 100 & 6.023 & $3 \cdot 394$ & 2.629 \\
\hline
\end{tabular}

Observação: expoentes a, c, d = número de casos de associação parasitária da espécie com A. cajennense; b = número de casos de associação parasitária de A. cajennense com outra espécie.

Tabela 2. Percentual de humanos parasitados por diferentes espécies de carrapatos duros (Acari: Ixodidae), e associações de espécies, nas comunidades do Maciço da Pedra Branca e do Maciço da Tijuca, constatado durante pesquisa entre Janeiro/1997 e Dezembro/2007.

\begin{tabular}{cccccccc}
\hline Espécies & $\begin{array}{c}\boldsymbol{A} \text {. } \\
\text { aureolatum }\end{array}$ & A. cajennense & A.dubitatum & A. ovale & A. varium & $\begin{array}{c}\text { I. loricatus } \\
\text { sanguineus }\end{array}$ \\
\hline Total de casos & $176(2,92 \%)$ & $4.423(73,44 \%)$ & $201(3,34)$ & $46(0,76 \%)$ & $528(8,77 \%)$ & $9(0,15 \%)$ & $640(10,63 \%)$ \\
Associada a & $\mathrm{A}_{20}$ & 4.423 & 8 & 0 & 0 & 0 \\
$\begin{array}{c}\text { Amblyomma } \\
\text { cajennense }\end{array}$ & ${ }^{\mathrm{B}} \mathrm{O}, 33 \%$ & $73,44 \%$ & $0,13 \%$ & 0 & $0,84 \%$ \\
\hline
\end{tabular}

A. Número de casos; B. Percentagem sobre o número de casos de ixodidose; C. Percentagem sobre o número de casos com a espécie. 
humanos têm sido relatados no Brasil (Famadas et al. 1997; MARques et al. 2006; Labruna et al. 2007; Soares et al. 2007; FERREIRA et al. 2008). Ficou destacado que jovens e crianças são mais vulneráveis (Figura 2). Para as cerca de 115.000 pessoas que integram a população que co-habita, trabalha ou explora o espaço investigado, o coeficiente de prevalência de ixodidose calculado foi de 5,24\%. Considerando as faixas etárias relacionadas ao sexo do hospedeiro, foi observado que entre jovens e adultos as prevalências por sexo se equivalem, pois a probabilidade do sexo estar influenciando o parasitismo teve diferença não significativa $(\mathrm{p}>0,05)$.

Aconteceram casos com todos os estágios evolutivos, concomitantes ou não, além de ter havido parasitismo só por uma espécie, ou parasitismo simultâneo por duas espécies de carrapato, confirmando achados de Serra-Freire (2010). Estes casos foram entre A. cajennense e uma destas três: Rhipicephalus sanguineus (Latreille), Amblyomma aureolatum (Pallas) ou Amblyomma dubitatum Neumann (Figura 2). Só foram identificadas larvas de A. cajennense e A. varium. Também foram encontradas ninfas destas espécies, e de $R$. sanguineus. De Amblyomma ovale Kock só fêmeas foram encontradas; de A. aureolatum, A. cajennense, A. dubitatum, $R$. sanguineus, e Ixodes loricatus Neumann foram identificados machos e fêmeas parasitando humanos. A diversidade encontrada, incluindo espécies tidas como de ambiente silvestre, como $A$. aureolatum, A. ovale, e Amblyomma varium Kock reforçam a afirmação de Labruna (2004) sobre possibilidade de serem vetores de bioagentes de zoonoses emergentes. Pode ser o caso de $R$. rickettsii agente da Febre Maculosa Brasileira, transmitida por espécies de carrapatos de animais silvestres (ARAGÃo 1936; Lemos et al. 1996; SouZA et al. 2006; GAZÊTA et al., 2009), com suspeita, mas ainda não confirmada no espaço estudado.

A análise temporal dos achados dos onze anos evidenciou sazonalidade, caracterizada por semestralidade, sendo o primeiro semestre o de maior freqüência de casos, e o trimestre fevereiroabril o de maior incidência de achados (Figura 3). Entretanto não deve ser esquecido que o ciclo biológico pode variar entre 3 a 12 meses, e os adultos podem sobreviver por 5 a 12 anos, incluindo longos períodos de jejum (Flechtmann 1973; SerRaFreire \& Miziara 1989) o que pode mascarar esta observação sazonal quando investigada em curto período. A média do número mensal de casos de ixodidose humana foi de $51 \pm 12$, com o menor número de $35 \mathrm{em}$ agosto/1998, e maior de $80 \mathrm{em}$ março/2007 O cálculo da tendência secular mostra crescimento contínuo da incidência de casos (Figura 4), sendo que a média anual de casos de ixodidose humana foi de 548, sendo 1997 o ano de menor incidência comprovada com 195 casos, e o de maior incidência o de 2007, com 837 registros. Esta tendência preocupa principalmente se reunirmos as informações que as espécies dominantes entre as agora encontradas são comprovados vetores de agentes infecciosos para o humano e outros animais (GEHRKE et al. 2009)

A diversidade de espécies de carrapato duro foi similar entre os anos (CSs = 89,9), tendo sido a maior diferença o encontro de I. loricatus somente nos dois últimos anos da pesquisa.

$\mathrm{O}$ encontro de $R$. sanguineus parasitando humanos confirma que esta relação acontece no Brasil, como demonstram os artigos de Louly et al. (2006) em Goiás, Dantas-TorRes et al. (2006) em Pernambuco, Serra-Freire (2010) no Pará e Venzal et al. (2003) no Uruguai, ou o risco de acontecer no Rio de Janeiro (SERra-Freire \& Borsol 2009). Foi confirmado que A. cajennense é a espécie de carrapato duro dominante entre as que parasitam humanos no Brasil, e América do Sul (EstradA-Peña \& Jongejan 1999; Massard \& Fonseca 2004; Onofrio et al. 2006; Serra-Freire 2010). Os resultados também confirmam que A. varium pode parasitar humanos, como assinalaram АмоRiм et al. (2003).

O parasitismo por A. aureolatum, A. dubitatum e A. ovale, agora registrados não é muito relatado na literatura (Guglielmone et al. 2006), ou talvez não seja mesmo muito comum, e estas espécies já são conhecidas no espaço geopolítico do Rio de Janeiro há muito tempo (ARagão 1936; Aragão \& FonseCA
1961). É necessário considerar que A. aureolatum e I. loricatus já foram assinalados como parasitos de gambá (Didelphis albiventris Lund) que coloniza o peridomicílio (MulLer et al. 2005), favorecendo por aproximação o encontro de humanos por estas espécies de carrapatos.

O encontro de maior percentual de homens parasitados por carrapatos, em relação às mulheres (Figura 2), em todas as classes de faixa de idade, assim como o fato de que os grupos de crianças e jovens foram os de maiores números de casos, parece ser decorrência da maior exposição destes grupos de pessoas aos fatores condicionantes. Esta linha de raciocínio se identifica as de Louly et al. (2006) e SerRA-Freire (2010), encontrando base na fraca organização social daquela comunidade, pouca infra-estrutura de saneamento básico, e grande contato entre os animais e os humanos. A ocupação do espaço de forma desordenada e contínua, sem planejamento urbano, confunde os nichos e condiciona ao aumento da relação simbiótica prejudicial para os coabitantes locais, como fica evidenciado pela tendência secular crescente do número de casos (Figura 4). Todos os meses do ano ocorrem casos de ixodidose humana, podendo ser dito que esta doença está enzoótica na comunidade, com limiar enzoótico igual ao coeficiente de prevalência ( $\mathrm{CP}=5,24 \%)$, com sazonalidade presente, mas pouco marcante (Figura 3). Não se conseguiu uma explicação para só ter sido encontrado associação parasitária com A. cajennense.

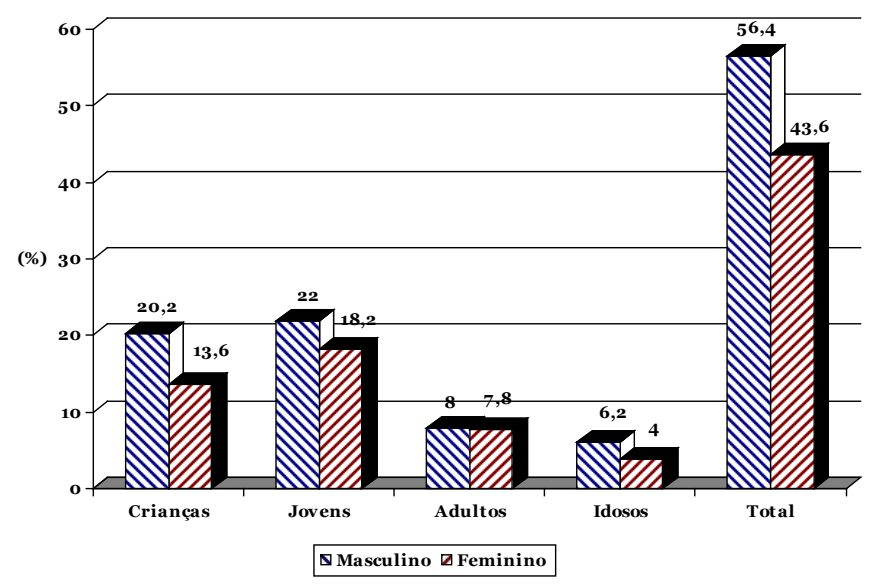

Figura 2. Coeficiente de prevalência, por faixa etária e sexo, para humanos infectados por carrapatos duros (Acari: Ixodidae), entre os 6.023 hospedeiros identificados nas comunidades do Maciço da Pedra Branca e do Maciço da Tijuca, durante pesquisa entre Janeiro/1997 e Dezembro/2007.

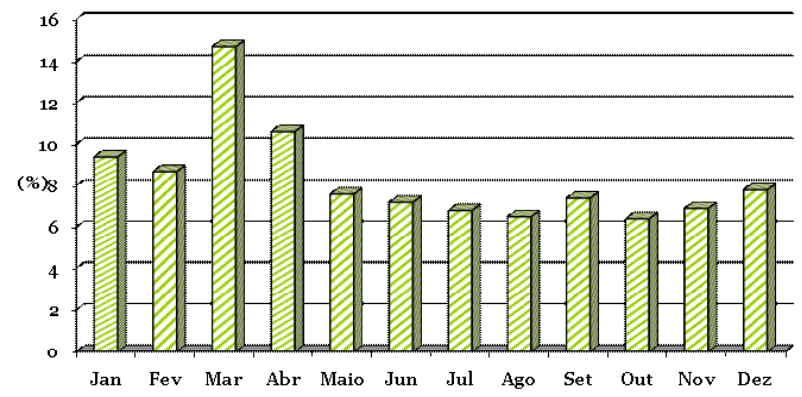

Figura 3. Frequência mensal de casos de ixodidose humana por carrapatos duros, entre os 6.023 hospedeiros parasitados, nas comunidades do Maciço da Pedra Branca e do Maciço da Tijuca, constatados entre Janeiro/1997 e Dezembro/2007. 


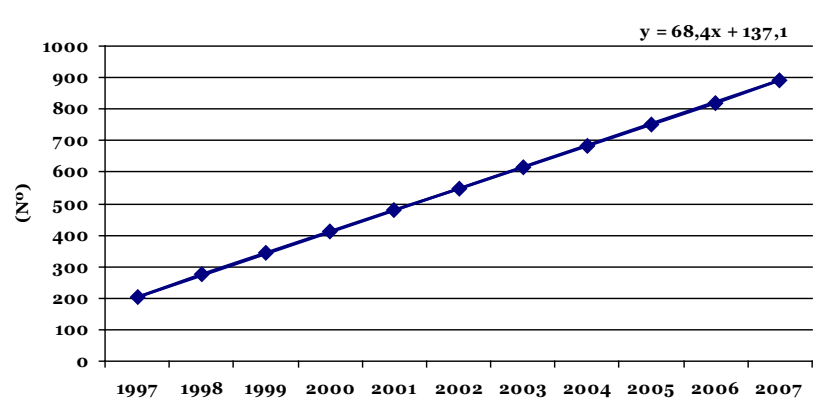

Figura 4. Tendência secular de casos de ixodidose humana, por carrapatos duros entre ocupantes das comunidades do Maciço da Pedra Branca e do Maciço da Tijuca, Rio de Janeiro, RJ, Brasil.

\section{REFERÊNCIAS}

Amorim, M \& N.M. Serra-Freire, 1999. Chave dicotômica para identificação de larvas de algumas espécies do Gênero Amblyomma Koch, 1844 (Acari: Ixodidae). Entomologia y Vectores, 6: 75-90.

Amorim, M., R.H.F. Teixeira, M.P. Valim, G.S. Gazêta \& N.M. Serra-Freire, 2003. Espécies de carrapatos (Acari: Ixodidae) parasitando humano no zoológico de São Paulo, São Paulo. Revista da Sociedade Brasileira de Medicina Tropical, 36: 232-233.

Aragão, H.B., 1936. Ixodidas Brasileiros e de alguns países limitrophes. Memórias do Instituto Oswaldo Cruz, 31: 759845 .

Aragão, H.B. \& F. Fonseca, 1961. Notas de ixodologia VIII listachave para os representantes da fauna ixodológica brasileira. Memórias do Instituto Oswaldo Cruz, 59: 115-129.

Barbieri, F.S., 2005. Quetotaxia e porotaxia das larvas de Amblyomma spp. (Acari: Ixodidae) do Brasil, e descrição de larva de Amblyomma ovale Koch, 1844. Tese de doutorado, UFRRJ, 77p.

Barros-Battesti, D., M. Arzua \& G.H. Bechara, 2006. Carrapatos de Importância Médico-Veterinária da Região Neotropical: um guia ilustrado para identificação de espécies. São Paulo: VOX, 223p.

Boero, J.J., 1957. Las garrapatas de La Republica Argentina (Acarina: Ixodoidea). Departamento Editorial Universidad de Buenos Aires, 113p.

Clifford, C.M. \& G. Anastos, 1960. The use of chaetotaxy for the identication of larval ticks (Acari: Ixodidae). Journal of Parasitology, 46: 567-578.

Cooley, R.A \& G.M. Kohls, 1944. The genus Amblyomma (Ixodidae) in the United States. Journal of Parasitology, 30: 77-111.

Cunha, N.C., A.H. Fonseca, J. Rezende, T. Rozental, A.R.M. Favacho, J.D. Barreira, C.L. Massard \& E.R.S. Lemos, 2009. First identification of natural infection of Rickettsia rickettsii in the Rhipicephalus sanguineus tick, in the State of Rio de Janeiro. Pesquisa Veterinária Brasileira, 29: 105-108.

Dantas-Torres, F., L.A. Figeuredo, S.P. \& Brandão-Filho, 2006. Rhipicephalus sanguineus (Acari: Ixodidae), the Brown dog tick, parasitizing humans in Brazil. Revista da Sociedade Brasileira de Medicina Tropical, 39: 64-67.

Estrada-Peña, A. \& F. Jongejan, 1999. Ticks feeding on humans: a review of records on human-biting Ixodoidea with special reference to pathogen transmission. Experimental Applied Acarology, 23: 685-715.

Famadas, K., E.R.S. Lemos, J.R. Coura \& N.M. Serra-Freire, 1997. Amblyomma cooperi (Acari: Ixodidae) parasitando humano em área de foco de febre maculosa, São Paulo, Brasil. Acta Parasitológica Portuguesa, 4 (sup. 1): 154.

Ferreira, C.G.T., I.G. Rego \& S.M.M. Ahid. 2008. Parasitismo em humano por Amblyomma parvum Aragão, 1908 (Acari: Ixodidae) em Pau dos Ferros, Rio Grande do Norte, Brasil. Publicações em Medicina Veterinária, 3: 1-6.
Filippova, N. A. 1984. Taxonomy of ticks of the family Ixodidae (Acarina, Parasitiformes) in the fauna of the USRR and plans for studying it. Paraziyologicheskii sbornik, 32: 61-78.

Flechtmann, C.H.W., 1973. Acarologia de interesse Médico Veterinário, São Paulo: Nobel, 122p.

Gazêta, G.S., E.R. Souza, A.E. Abboud-Dutra, M. Amorin., P.R. Barbosa., A.B. Almeida, V. Gomes, F.S Gehrke, M.T. Marrelli \& T.T.S. Schumaker, 2009. Potential vectors and hosts of Rickettsia spp: epidemiological studies in the Vale do Paraíba, state of Rio de Janeiro/Brazil. Clinical Microbiology and Infection, 15: 269-270.

Gehrke F.S., G.S. Gazêta, E.R. Souza, A. Ribeiro, M.T. Marrelli \& T.T.S. Schumaker, 2009. Infecting Rhipecephalus sanguineus and Ctenocephalides felis collected from dogs in a Brazilian spotted fever focus in the State of Rio de Janeiro/Brazil. Clinical Microbiology and Infection, 15: 267-268.

Guglielmone, A.A., L. Beati, D.M. Barros-Battesti, M.B. Labruna., S. Nava, J.M. Venzal, A.J. Mangold, M.P.J. Szabó, J.R.S. Martins., D. Gomzáles-Acuna \& A. Estrada-Peña, 2006. Ticks (Ixodidae) on humans in South America. Experimental and Applied Acarology, 40: 83-100.

Hoogstraal H., A. Aeschlimann, 1982. Tick-host specificity. Bulletin de le Societé Entomologique Suisse, 55: 5-32.

Jones, E.K., C.M. Clifford \& J.E. Keirans. 1972 The ticks of Venezuela (Acari: Ixodidae) with a key to the species of Amblyomma in the western hemisphere. Brighan Young University Science Bulletin Biological Serie, v. 17.

Keirans, J. E \& L.A. Durden, 1998. Illustrated key to nymphs of the tick genus Amblyomma (Acari: Ixodidae) found in the united state. Journal of Medical Entomology, 35: 489-495.

Labruna, M.B., 2004. Carrapatos. A Hora Veterinária, 137: 6365.

Labruna M. B., R.C. Pacheco, A.C. Ataliba \& M.P.J. Szabó, 2007. Human parasitism by the capybara tick Amblyomma dubitatum (Acari: Ixodidae). Entomological News, 118: 7780.

Lemos, E.R.S., H.H.B. Melles, S. Colombo, R.D. Machado, J.R. Coura, M.A.A. Guimarães, S. Sanseverino \& A. Moura, 1996. Primary isolation of spotted fever group rickettsiae from Amblyomma cooperi collected from Hydrochaeris hydrochaeris in Brazil. Memórias do Instituto Oswaldo Cruz, 91: 273-275.

Louly, C.C.B., I.N. Fonseca, V.F. Oliveira \& L.M.F. Borges, 2006. Ocorrência de Rhipicephalus sanguineus em trabalhadores de Clínicas Veterinárias e Canis, no Município de Goiânia, Go. Ciência Animal Brasileira, 7: 103-106.

Marques, S., R. Dal- Col, M.O. Matos Júnior, E.F. Gonçalves, E.F, A. Pinter \& M.B. Labruna, 2006. Parasitismo de Amblyomma fuscum (Acari: Ixodidae) em humanos. Ciência Rural, 36: 1328-1330.

Massard, C.L \& A.H. Fonseca, 2004. Carrapatos e doenças transmitidas, comuns ao homem e aos animais. A Hora Veterinária, 137: 15-23.

Muller, G., J.G.W. Brum, P.Q. Langoni, G.H. Michels, A.L. Snkoc, J.L. Ruas \& M.E.A. Berne, 2005. Didelphis albiventris Lund, 1841, parasitado por Ixodes loricatus Neumann, 1899, e Amblyomma aureolatum Pallas, 1772, (Acari: Ixodidae) no Rio Grande do Sul. Arquivos do Instituto Biológico, 72: 19324.

Oliver Jr, J.H. 1989. Biology and systematic of ticks (Acari: Ixodidae). Annual Review of Ecology and Systematics, 20: 397-430.

Onofrio, V.C., M.B. Labruna, A. Pinter, F.G. Giacomin, D.M. Barros-Battesti, 2006. Comentários e chaves para as espécies do gênero Amblyomma, p. 53-113. In: Barros-Battesti, D.M., M. Arzua \& G.H. Bechara. Carrapatos de importância médico veterinária da região neotropical: um guia ilustrado para identificação de espécies. São Paulo: VOX. 224 p.

Pinter A., M.C. Horta \& M.B. Labruna. Febre Maculosa informe técnico II: vigilância epidemiológica. Disponível em: 
$<$ ftp://ftp.cve.saude.sp.gov.br./doc tec/ZOO/IF FMB2. pdf $>$. Acesso em: 21 fev. 2008.

Robinson, L.E., G.H.F. Nutall \& C.L.E. Warburnon, 1926. The genus Amblyomma in Ticks: a monograph of the Ixodidae. Part IV. London. Cambridge University Press, p. 302.

Serra-Freire, N.M., 2001. Ácaros: carrapatos e outros. In: Marcondes, C.B., Entomologia Médica Veterinária. São Paulo: Atheneu, VII+432p.

Serra-Freire, N.M., 2010. Occurrence of ticks (Acari: Ixodidae) on human hosts, in three municipalities in the State of Pará, Brazil. Brazilian Journal Veterinary Parasitology. 19: 1-8.

Serra-Freire, N.M., 2002. Planejamento e análise de pesquisas parasitológicas. Niterói-RJ: EdUFF - Universidade Federal Fluminense, $199 \mathrm{p}$.

Serra-Freire, N.M., 1983. Tick paralysis in Brazil. Tropical Animal Health and Production, 15: 124-126.

Serra-Freire, N.M \& A.B.P. Borsoi, 2009. Malformação em teleógina de Rhipicephalus sanguineus recolhida em ambiente intradomiciliar no Rio de Janeiro, RJ. Revista Brasileira de Parasitologia Veterinária, 18: 55-58.

Serra-Freire, N.M \& S.R. Miziara, 1989. Influência do hospedeiro no ciclo e comprovação do ciclo heteroxeno de Anocentor

Como citar este artigo:

N.M. Serra-Freire, L.M.M. Sena \& A.B.P. Borsoi, 2011. Parasitismo Humano por Carrapatos na Mata Atlântica, Rio de Janeiro, Brasil. EntomoBrasilis, 4(2): 67-72. www.periodico.ebras.bio.br/ojs nitens (Neumann, 1897). Memórias do Instituto Oswaldo Cruz, 84: 213-218.

Soares, J.F., L.A. Sangioni, F.S.F. Vogel \& F.B. Silva, 2007. Parasitismo em ser humano por B. microplus (Acari: Ixodidae) em Santa Maria, RS, Brasil. Ciência Rural, 37: 1495-1497.

Sonenshine, D.E. 1991. Biology of ticks. Oxford: Oxford University Press. v.1. 446p.

Souza, S.S.A.L., C.E. Souza, E.J. Rodrigues-Nweto \& A.P. Prado., 2006. Dinâmica sazonal de carrapatos (Acari: Ixodidae) na mata ciliar de uma área endêmica para febre maculosa na região de Campinas, São Paulo, Brasil. Ciência Rural, Santa Maria, 36: 887-891.

Venzal, J.M., A.A. Guclielmont, A. Estrada-Pen, P.A. Cabrera \& O. Castro, 2003. Ticks (Ixodida: Ixodidae) parasitising humans in Uruguay. Annals of Tropical Medicine and Parasitology, 97: 769-772.

Recebido em: 30/06/2010

Aceito em: 12/08/2010

$* * * * * * * * * * * * *$
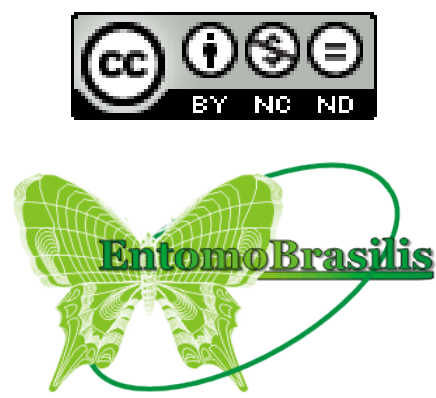